\title{
Stimulation of the Dorsal Premotor Cortex, But Not of the Supplementary Motor Area Proper, Impairs the Stop Function in a STOP Signal Task
}

Sara Parmigiani ${ }^{\mathrm{a} *}$ and Luigi Cattaneo ${ }^{\mathrm{b}}$

a Department of Biomedical and Clinical Sciences "L. Sacco" and Department of Pathophysiology and Transplantation, University of Milan, via G. B. Grassi 74, 20157 Milano, Italy

b Department of Neurosciences, Biomedicine and Movement Sciences, University of Verona, Strada Le Grazie 8, 37143 Verona, Italy

\begin{abstract}
Being able to inhibit an impending movement in response to a contextual change is a distinctive feature of action control. Such inhibitory control relies on a complex cortical-subcortical network, including posterior prefrontal regions such as caudal inferior frontal gyrus and pre-supplementary motor area. According to hierarchical models of action control, both areas represent the intermediate level between prefrontodependent and motor-related cortices. Going at a lower level, accumulating evidence speaks for an involvement of the primary motor cortex (M1) to dorsal premotor cortex (PMCd) or supplementary motor area proper (SMAproper) pathways in producing inhibitory control. However, the clear-cut evidence for this conjecture is still missing. The aim of the present paper was to start filling this gap, investigating this lowest level of inhibitory control. We stimulated PMCd in a group of healthy volunteers with transcranial magnetic stimulation (TMS) or sham TMS during the response phase of a STOP-signal task performed with the lips. In a separate experimental group, we applied effective TMS/ sham TMS to SMA-proper during the same task. We found that effective TMS over PMCd increased false-start errors in STOP trials $(p=0.0005)$, but had no effect on GO trial performance ( $p$ $=0.85)$. Effective TMS on SMAproper produced no effect on STOP trials' performance $(p=0.31)$ nor in the GO trial performance $(p=0.56)$. Our data show that there is at least a portion of PMCd playing a distinctive role in the control of mouth-related M1 during instructed visuomotor inhibitory behavior. This region could therefore represent a low-level hierarchical node for externally cued action inhibition. 2018 IBRO. Published by Elsevier Ltd. All rights reserved.
\end{abstract}

Key words: premotor cortex, supplementary motor area, executive control, inhibition, motor control.

\section{INTRODUCTION}

Being able to inhibit an impending movement in response to a contextual change is a distinctive feature of action control. The caudal inferior frontal gyrus (cIFG) and the pre-supplementary motor area (pre-SMA) have been identified as the two pivotal cortical nodes in domaingeneral response suppression. Authoritative models postulate a hierarchical gradient of abstractness in executive functions, partly, but not entirely corresponding to an anatomical gradient in the rostrocaudal direction of the frontal lobes (Badre and D'Esposito, 2009; Caminiti et al., 2017). The premotor cortex (PMC) also seems to follow a rostro-caudal hierarchical principle. In monkeys, anterior premotor regions have an exclusive connection to the prefrontal cortex and little direct access to the primary motor cortex (M1), and were described as prefronto-dependent regions (Rizzolatti and Luppino, 2001). Conversely, the caudal premotor regions are densely interconnected with the parietal cortex and exert strong direct inputs to M1 and were described as parieto-dependent regions (Muakkassa and Strick, 1979; Tokuno and Tanji, 1993; Kiefer et al., 1998; Hatanaka et al., 2001). Within this framework, the pre-SMA (Brodmann's area 6 - BA6) and the right caudal IFG (BA44) can be categorized indifferently as high-level (prefronto-dependent) PMCs, or as low-level prefrontal cortices, in that neither pre-SMA nor the right cIFG have direct access to the motor output in $\mathrm{M} 1$ and contain abstract effector-and domainindependent action representations. On one side, there is a well-known low-level inhibitory pathway passing though the somatosensory cortex: the short-latency afferent inhibition (SAI) is involved in the inhibition of unwanted muscle contractions during action via direct projections to M1 (Voller et al., 2006; Asmussen et al., 2013, 2014). 
Moreover, SAl has been shown to be sensitive to cognitive functions, as attentional load (Mirdamadi et al., 2017). On the other side, there is a missing link in the chain of information from the prefronto-dependent regions and the motor cortex via premotor pathway. It is hypothesized that the inhibitory control is exerted via the caudal premotor cortex (Dum and Strick, 1991, 2005), but no direct evidence of a relay function of the PMC in action inhibition is available in humans. In other words, there is no clear evidence of inhibitory connections from the caudal PMC to M1 that are functional to stopping behavior. Currently, there are neurostimulation as well as clinical data pinpointing at two caudal PMC regions, the dorsal premotor cortex (PMCd) and SMA-proper, that could represent low-level inhibitory nodes (Nii et al., 1996; Lu“ ders et al., 1998; Mikuni et al., 2006; Duque et al., 2012, 2017; Filevich et al., 2012) giving origin to inhibitory projections to M1.

As far as PMCd is concerned, cortico-cortical connectivity between left PMCd and the contralateral M1, assessed by dual-coil transcranial magnetic stimulation (TMS), was found to be inhibited when prepared actions should be suppressed (Koch et al., 2006) and a previous study has shown that offline neuromodulation of PMCd specifically impacts inhibitory behavior (Duque et al., 2012). In this direction, we have recently described direct evidence of task-dependent inhibitory cortico-cortical connectivity from PMCd to mouth-related M1, in the form of a robust short-latency $(6 \mathrm{~ms})$ inhibitory effect of PMCd stimulation on ipsilateral corticobulbar excitability. Crucially, the inhibitory effects were manifest when the contextual information prompted immobility (Parmigiani et al., 2015, 2018). As for SMA-proper, a caudal premotor region located adjacent to PMCd on the medial surface of the hemispheres and origin of consistent contingent of corticospinal fibers (Mayka et al., 2006), it has been associated with several "negative" or inhibitory motor phenomena in humans (Meletti et al., 2000; Krainik et al., 2004; Filevich et al., 2012).

Clear-cut causal evidence that these regions that mediate behavioral inhibition by projecting direct inhibitory control over M1 is still missing, and filling this gap is the main aim of the present work. We applied online eventrelated, single-pulse, sham or effective TMS to PMCd (Experiment 1) or SMA-proper (Experiment 2) in two different experiments and measured the performance of healthy volunteers in a Stop-signal task (SST) (Verbruggen and Logan, 2008; Noorani and Carpenter, 2017). Results show effective TMS impaired stopping of the ongoing response only when applied to PMCd and not to SMA. We describe causal evidence for a role in $\mathrm{PMCd}$ in reactive, externally cued action inhibition.

\section{EXPERIMENTAL PROCEDURES}

\section{Participants}

Twenty healthy individuals (15 women, mean age 24.2, ranging 20-29 years, SD 2.8) took part in Experiment 1. Fifteen different healthy individuals took part in Experiment 2 (11 women, mean age 23.8 years, ranging 19-29 years, SD 3.2). They all provided informed consent. They all were screened for any contraindication to TMS (Rossi and Hallett, 2009). The study was approved by the local ethics committee (protocol 2013-032) and was conducted in compliance with the revised Helsinki declaration [Helsinki World Medical Association, 2009]. The two experiments were identically structured, what changed between them was the site of TMS application. Participants were sitting comfortably on a chair with both chin- and forehead-rest. TMS was delivered in single pulses, in every trial, in an eventrelated fashion, time-locked to the GO-signal, at a time jittered over $200 \mathrm{~ms}$ just prior to the timing of the eventual STOP-signal (the STOP-signal was not present in all trials, as in all SST). Responses were given with an upward movement of the lips and recorded by means of a custommade response apparatus. TMS was delivered in blocks in which either an effective coil or a sham coil was used. Summing up, both Experiment 1 and Experiment 2 were organized according to a factorial within-subjects design with the factors: TMS (2 levels: effective or sham); TASK (2 levels: GO trials or STOP trials). The total number of trials was 480, divided equally in the four cells of the 22 experimental design. The TASK was randomized between trials, while the TMS factor was blocked. The trials were divided in 4 blocks, 2 of which were with sham TMS and the other 2 with effective TMS. 


\section{Apparatus for lip response collection}

All participants held a stick with their lips to collect the motor responses. To stabilize and ensure a constant orbicularis oris (OOr) contraction throughout the experiment, a 15-g weight was suspended at the end of the stick. The actual motor response consisted in lifting the stick from a starting point until an end position was met, after which no more lifting was possible. At the instant when the end position was reached, a circuit was closed providing a $+5 \mathrm{~V}$ square-wave output. The output signal was transmitted to a PC via the serial port by the EPrime 2 software. The tip of the response stick contained also a 3-axe analog accelerometer, the output of which was constantly recorded throughout the trial length, by means of the EMG recording apparatus. The accelerometer's signal was analyzed offline to provide accurate estimation of the onset of the motor response, i.e. of reaction times (RTs). Response times indicated by E-prime were used uniquely for the display of singletrial feedback.

\section{Baseline performance for the definition of TMS and STOP-signal timing}

Before the experiment, participants were tested for their RTs in a simple reaction time task. After a variable inter-trial period (600-4500 ms), signaled by a black screen with colored stars, a GO-signal appeared in the center of the screen, informing the participants to perform a lifting of the stick, always kept between their lips, from the starting point to the arriving point. Participants were instructed to respond as quickly as possible to the GO-stimulus. They underwent at least 200 trials of this task. Then the values of RTs corresponding to the first (T1), second (T2) and third (T3) decile of the single subject's RTs' distribution were extracted and used to define the timing of the TMS pulses and the onset of the STOP-signal. TMS was jittered (uniform distribution along a square wave) between the individuals T1 and T3, and the STOPsignal, when present, was systematically presented at the fixed time corresponding to $\mathrm{T} 2$. Also the singlesubject single-reaction time task 7 th decile (T7) was computed and used later as a parameter in the main task. In case the subjects used more time than their T7 of the distribution of their RTs, at the end of the trial they received a proper feedback (see Behavioral task paragraph). The 9th decile (T9) was used as the maximum time to wait for a response in GO trials. Fig. 1 illustrates the individual values of each timing in the two experiments.

\section{Behavioral task}

Fig. 2 illustrates the trial structure in the two main experiments. The SST was composed of a combination of $50 \%$ of GO and $50 \%$ of STOP trials (60 GO and 60 STOP trials). In all trials, a GO-signal was presented at the beginning of the trial (TO), to which participants had to respond as quickly as possible with the mouth movement. In STOP trials, a STOP-signal appeared at a fixed time after the GO-signal based on individual baseline performance (T3, the stop signal delay - SSD). The appearance of the STOP-signal informed the participants to arrest their response (Obeso et al., 2013). On-line feedback informed participants if they successfully accomplished the GO or STOP trials (Good!) or failed (Wrong!). In case they took more time than their individual T7 time, they were "pushed" to not slow down with an appropriate feedback (Warning! You are too slow!), on the contrary, in case they did not wait for the GO-signal to occur, they were warned to do not anticipate

(Don't anticipate!). GO trials were considered as errors if the subject had not given any response by the time of T9. By providing such feedbacks we expected to minimize unwanted strategies such as a) always responding, b) never responding, or c) waiting after T3 to know whether a trial is a STOP or a GO trial to prepare the response. The inter-trial interval was variably jittered between 2100 and $4500 \mathrm{~ms}$. 

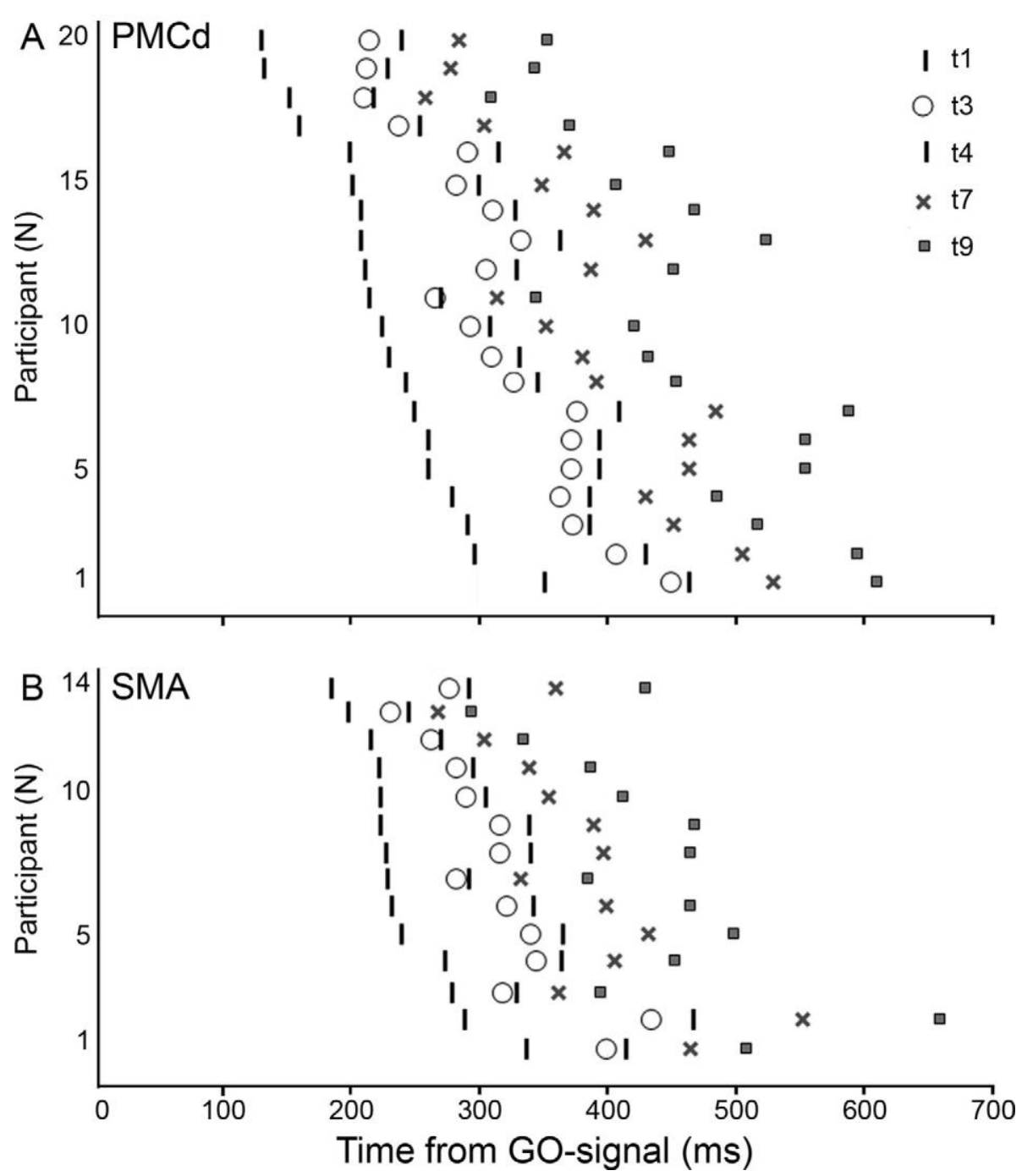

Fig. 1. Illustration of timings used in the experiment for each of the participants, ordered by average RT. The single time points are calculated on the basis of percentiles of individual RTs during the simple reaction time task. $T 1=$ first decile; $t 3=$ third decile; $t 4=$ fourth decile; $t 7=$ seventh decile; $t 9=$ ninth decile.

\section{TMS}

During the response period, singlepulse TMS was delivered over PMCd (Experiment 1) or SMAproper (Experiment 2) after the occurring of the GO-signal. Stimulation timing was jittered around the STOP-signal at random times distributed following a square wave between T1 and T3. In two of the four blocks participants received effective TMS and in the other two they received sham TMS. The order of stimulation was pseudorandomized across subjects. TMS intensity was dosed as a function of resting motor threshold (RMT). Therefore, in a preliminary step we recorded EMG of the right first dorsal interosseous (1DI) on which RMT was calculated in Experiment 1. Conversely, in Experiment 2, considered that the SMA-proper target lies deeper than the PMCd target, we assessed RMT relative to the EMG recordings of the right tibialis anterior (TA) muscle. The intensity of the stimulation was around $100 \%$ of the RMT of the right 1DI muscle in PMCd experiment and at $100 \%$ of the RMT of the right TA muscle in SMA experiment. These choices are due to the difference in the $z$ Talairach dimension between PMCd and SMA. Since the rationale of using the RMT for non-primary motor areas 
rely on the assumption that the convexity of the cortex should be the same of the hand $M 1$, and in our case this is true for PMCd only, we moved to a same-z dimension M1, namely the leg-relate M1 (see Gerloff et al., 1997). The leg representation in the M1 has the same $z$ and $x$ coordinates with respect to the SMA (the only difference concerns the $x$ coordinate). Indeed, in clinical use of TMS over SMA-proper, the individual intensity is commonly scaled on the foot/leg motor threshold (see, for instance, Hamada et al., 2008). Singlepulse biphasic stimulation was achieved by means of a MagPro R100 biphasic magnetic stimulator (Medtronic, Denmark) connected to a MCFB65 coil with 65-mm windings (MagVenture, Skovlunde, Denmark) for effective stimulation. Sham stimulation was achieved with a MCF-P-B65 coil with 65-mm windings placebo figure-ofeight coil (MagVenture, Skovlunde, Denmark). Coils were held by an articulated mechanical arm (Manfrotto 244, VitecGroup, Italy). The PMCd coil was positioned with a medio-lateral orientation of the induced current. The SMA-proper coil was positioned tangentially to the midline with the handle positioned caudally.

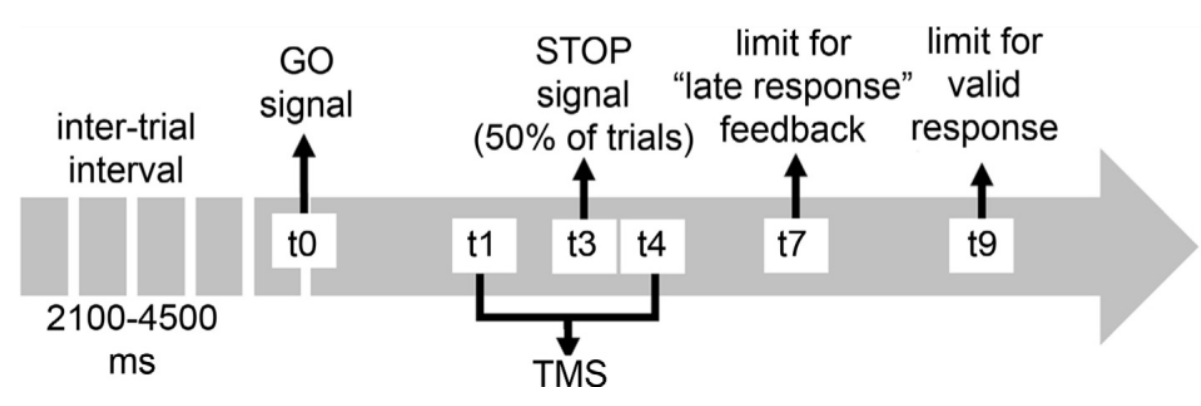

Fig. 2. Structure of a single trial of Experiments 1 and 2

\section{Neuronavigation and localization of TMS targets}

Neuronavigated-TMS was used and the targeted areas were localized by means of individual investigations of anatomical landmarks. Before the experimental session, a high-resolution T1-weighted magnetization prepared rapid gradient echo sequence (176 axial slices, in-plane resolution $2563224,1 \mathrm{~mm}$ isotropic voxels) scan of the brain of each subject was obtained, using a MedSpec 4 T head scanner (Bruker BioSpin GmbH, Rheinstetten, Germany) with an 8-channel array head coil. Starting from this scan, a 3D reconstruction of the scalp and the gray matter surfaces was produced using MesH morphing tool included in the BrainVoyager software (Brain Innovation BV, The Netherlands). The BrainVoyager neuronavigation software combined with an ultrasound tracking system, CMS205S (Zebris Medical GmbH, Isny, Germany), was used to coregister the 3D scalp reconstruction with the actual participant's head, thus marking the target point for the conditioning TMS on the real head of each participant. In Experiment 1, TMS was delivered to the left mouth-related PMCd as defined in Parmigiani and colleagues (Parmigiani et al., 2015). In Experiment 2, TMS was delivered to the face/mouthrelated left SMA-proper. To localize this area, we took advantage of the review by Picard and Strick (Picard and Strick, 1996, but see also Vorobiev et al., 1998), in which they summarized the results of several positron emission tomography (PET) studies that examined functional activation on the medial wall of humans aimed to providing a common frame of reference for studies of medial wall function (medial portion of BA 6). According to this map, the AC projection crosses exactly in the middle the face area of pre-SMA and SMA-proper and therefore to identify this area we used an AC-PC translation of the brain MRIs from each participant and stimulated 2 millimeters caudally to the AC projection on the scalp (see Fig. 3).

\section{Statistical analysis}

TMS was delivered during the response time, and therefore, in a limited number of trials, subjects were particularly fast and TMS was delivered after the RT. These trials were $7.15 \%$ of the total trials of the Experiment 
1 and $6.1 \%$ of the Experiment 2 and were excluded from further analysis. Interestingly, excluded trials were almost equally distributed between GO and STOP trials. The main experimental variable was the accuracy. RTs are reported but were obviously not representative of STOP trials. Accuracy data from the two experiments were analyzed together in a mixed, between and within-subjects design. We performed a factorial withinsubject ANOVA with two within-subject factors: TMS (2 levels: effective or sham) and TASK (2 levels: GO-trials or STOP trials), and one betweensubject factor: TARGET ( 2 levels = PMCd or SMAproper). The post-hoc analyses were systematically performed by confronting effective TMS with sham TMS in every condition and correcting the results for the appropriate number of multiple comparisons.

\section{RESULTS}

None of the subjects reported undesired effects of TMS in both the experiments. As a preliminary assessment of the population's performance level in the SST, we calculated accuracy only in the SHAM condition, so that any effect of TMS was ruled out. Accuracy in GO trials corresponded to 71\% (ranging 66-92\%, SD 6.8) and in STOP trials it corresponded to $78 \%$ (ranging 69-94\%, SD 5.6). Considering that random behavior would have produced a chance-level accuracy of $50 \%$, the performance in our population was optimal for assessing any deviation in both directions of the accuracy of both STOP and GO trials. The mean of stimulation intensity was $59 \%$ of the stimulator output (range $46-77 \%$, SD 9.5) in Experiment 1 and $78 \%$ of the stimulator output (range $60-90 \%$ SD 0.5) in Experiment 2. The ANOVA showed a main effect of $\operatorname{TASK}\left(F(1,33)=6.19, p=0.018 ; g^{2}=0.16\right)$, a main effect of TMS $\left(F(1,33)=5.89, p=0.020 ; g^{2}=0.15\right)$ and a TARGET*TASK interaction $(F(1,33)=14.54, p=$ $\left.0.0006 ; g^{2}=0.31\right)$. The most important result was a significant 3-way TARGET*TASK*TMS interaction $(F(1,33)=$ 4.2936, $\mathrm{p}=0.046 ; \mathrm{g}^{2}=0.12$ ). The interaction was investigated by paired $\mathrm{t}$-tests between the sham and the effective TMS conditions within the same factors: (a) PMCd stimulation - STOP trials: sham TMS was different from effective TMS $(\mathrm{t}(19)=4.20, \mathrm{p}=0.0005)$; (b) PMCd stimulation $-\mathrm{GO}$ trials: sham TMS was not different from effective TMS $(\mathrm{t}(19)=0.19, \mathrm{p}=0.85)$; (c) SMA stimulation $-\mathrm{STOP}$ trials: sham TMS was not different from effective TMS ( $t(14)=1.05, p=0.31)$; (d) SMA stimulation $-\mathrm{GO}$ trials: sham TMS was not different from effective TMS $(t(14)=0.59, p=0.56)$. The data are shown in Fig. 4.
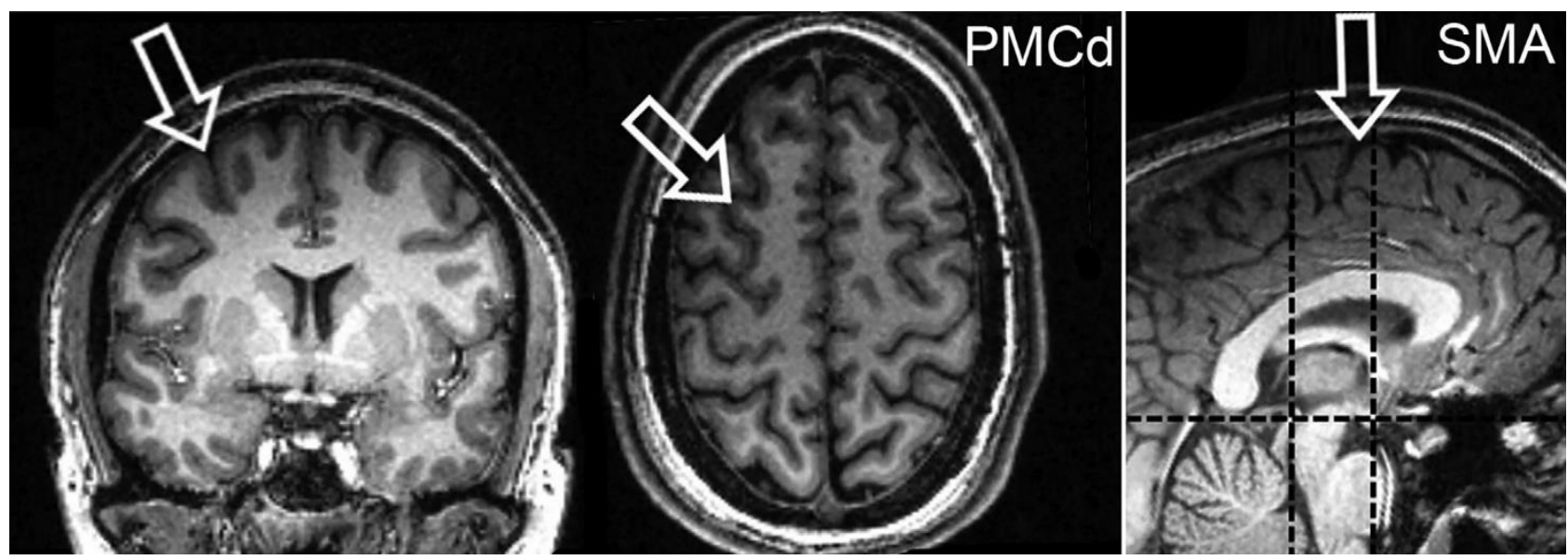

Fig. 3. Individual target localization in a representative subject (white arrows). From left to right, the PMCd target is shown in the first two figures, both in coronal and horizontal views, followed by the SMA-proper localization in sagittal view. The SMA-proper was identified taking advantage of the AC-PC coordinates. 


\section{DISCUSSION}

The present study aimed at investigating whether and to what extent the premotor cortices are involved in exerting cortical inhibition, mediating the ability to stop an ongoing action in response to a contextual change. Inhibitory areas with prefronto-dependent characterization, as CIFG or pre-SMA, should require a passage of inhibitory information through hierarchically lower regions that directly transmit it to $M 1$, with the lowest level cortical target likely represented by the PMCs. In our former work (Parmigiani et al., 2018), we tested the hypotheses that PMCd directly affects motor output by means of cortico-cortical projections to the ipsilateral mouth-related M1 and that the effect of such connections is inhibitory whenever correct behavior requires immobility. We found that, when participants were performing the withholding phase of a delayed motor task involving the lip effector, conditioning with TMS the left PMCd lead to a time specific inhibition of the orofacial motor evoked potentials (MEP). This is an important evidence in the direction of an essential role of PMCd in action inhibition. However, only in presence of behavioral effects of TMS over PMCd - and over another comparable area associated with inhibitory motor phenomena such as SMA-proper - the physiological findings can be considered a strong argument for a distinctive role of PMCd in motor inhibition, i.e. representing the lowest level of inhibitory information transmission (see Hamani et al., 2004). To tackle these issues, we performed two experiments, in which we applied on-line, event-related, single-pulse TMS to the PMCd and SMA-proper and measured the accuracy of healthy volunteers performing a SST, compared to sham stimulation. There were two main findings. First, the effective stimulation of PMCd while participants were performing the action triggered by a GO-signal and countermanded by a STOP-signal produced an increase in errors compared to sham stimulation in the same condition. Importantly, this was true for STOP trials only. In the GO trials, performance was unaffected by TMS. Second, effective stimulation of SMA-proper produced no effect on STOP trials performance or on GO trial performance.

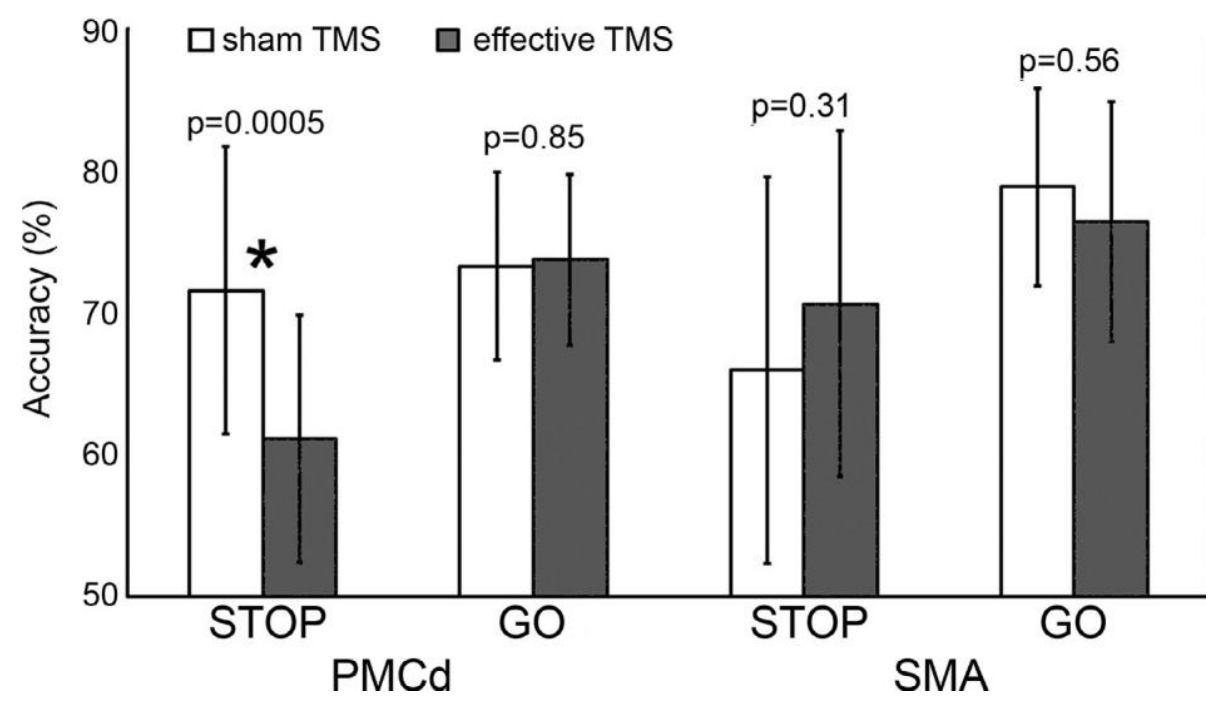

Fig. 4. Results of Experiment 1 (PMCd stimulation, $N=20$ ) and Experiment 2 (SMA-proper stimulation, $N=15)$. Accuracy is shown in percentage form $50 \%$ (chance level). White bars indicate level of accuracy while participants received the sham TMS, gray bars while they received the effective TMS. P-values indicate the results of paired $t$-tests between sham and effective TMS. Therefore, compared to sham stimulation, only the effective stimulation of PMCd in the STOP trials

\section{Supporting evidence for a role of PMCd in cued inhibition}

As far as this first finding is concerned, our results are in line with previous studies revealing an inhibitory role of PMCd in both non-human primates and humans. For example, Mirabella et al. recorded single neurons from 
PMCd of two monkeys performing both GO and STOP trials in a STOP-signal task (Mirabella et al., 2011). They showed that more than one third of recorded PMCd neurons involved in motor planning exhibit a countermanding modulation, with neurons that changed their pattern of discharge when a reaching movement was executed with respect to when it was inhibited. Kalaska and Crammond (Kalaska and Crammond, 1995) showed that neural activity of PMCd neurons changed when a movement should be suppressed with respect to when it should be executed. Similar results have been also reported by Ledberg (Ledberg et al., 2007) and colleagues, who showed that while the monkey was performing a GO/NOGO task, the cortical activity recorded in PMCd correlated with the decision to perform (or not) the response. This hypothesis seems also to be consistent with lesion studies: monkeys revealed increased frequency of impulsive and uncontrolled reaching movements following PMCd ablation (Moll and Kuypers, 1977) and loss of the ability to withhold movements after temporary inactivation of PMCd (Sawaguchi et al., 1996). In humans, stimulation of the premotor cortex causes "negative motor responses" characterized by the inability to perform certain voluntary movements or to sustain a voluntary muscle contraction. It has been shown that epileptic discharges in the premotor cortex could generate negative motor phenomena characterized by a loss in postural tone (Baumgartner et al., 1996; Meletti et al., 2000; Filevich et al., 2012). This seems to be in line with an electrical stimulation study in which were found negative motor responses following PMCd stimulation (Mikuni et al., 2006). Furthermore, humans with focal lesions, especially in the left superior portion of BA6, have been demonstrated to succumb to an increased number of false alarms, thus revealing a clear deficit in inhibiting responses to a stop signal (Picton et al., 2007). Supporting evidence comes also from TMS studies. Koch and colleagues (Koch et al., 2006) stimulated in a dual-coil paradigm the left PMCd and the contralateral M1 during a task in which participants responded with a hand squeeze to an auditory cue. The results showed coexistence of facilitatory and inhibitory drive from PMCd to M1 depending on whether the cue instructed to move the corresponding hand or to hold it still. Finally, Duque and colleagues (Duque et al., 2012) showed that repetitive TMS applied offline to the PMCd significantly diminished the characteristic suppression of MEP commonly observed during the set-period in a delayed reaction time task. It was recently proposed that inhibitory processes in PMCd are recruited in parallel with increasing preparatory activity (Li et al., 2016; Duque et al., 2017), generating inhibitory information targeting the M1, to prevent premature movements (Bizzi et al., 2000; Dum and Strick, 2005; Kroeger et al., 2010).

\section{Cautionary interpretation of the negative finding in SMA-proper}

Our second finding regards the substantial difference between PMCd and SMA-proper produced a significant increase in errors. activity of PMCd neurons changed when a movement should be suppressed with respect to when it should be executed. Similar results have been also reported by Ledberg (Ledberg et al., 2007) and colleagues, who showed that while the monkey was performing a GO/NOGO task, the cortical activity recorded in PMCd correlated with the decision to perform (or not) the response. This hypothesis seems also to be consistent with lesion studies: monkeys revealed increased frequency of impulsive and uncontrolled reaching movements following PMCd ablation (Moll and Kuypers, 1977) and loss of the ability to withhold movements after temporary inactivation of PMCd (Sawaguchi et al., 1996). In humans, stimulation of the premotor cortex causes "negative motor responses" characterized by the inability to perform certain voluntary movements or to sustain a voluntary muscle contraction. It has been shown that epileptic discharges in the premotor cortex could generate negative motor phenomena characterized by a loss in postural tone (Baumgartner et al., 1996; Meletti et al., 2000; Filevich et al., 2012). This seems to be in line with an electrical stimulation study in which were found negative motor responses following PMCd stimulation (Mikuni et al., 2006). Furthermore, humans with focal lesions, especially in the left superior portion of BA6, have been demonstrated to succumb to an increased number of false alarms, thus revealing a clear deficit in inhibiting responses to a stop signal (Picton et al., 2007). Supporting evidence comes also from TMS studies. Koch and colleagues (Koch et al., 2006) stimulated in a dual-coil paradigm the left PMCd and the contralateral M1 during a task in which participants responded with a hand squeeze to an auditory cue. The results showed coexistence 
of facilitatory and inhibitory drive from PMCd to M1 depending on whether the cue instructed to move the corresponding hand or to hold it still. Finally, Duque and colleagues (Duque et al., involvement in action inhibition during the SST. Participants in our study had no detectable behavioral change following SMAproper stimulation in any of the two experimental conditions (GO and STOP trials). The negative results of SMA-proper stimulation, as all negative results, have to be interpreted cautiously. They do not rule out the possibility that SMA-proper may contribute to action inhibition. On the contrary, they could be framed in a task-dependent perspective. One may say that the timing of the TMS used in our task does not cover the unfolding of the inhibitory pathway involving SMAproper. In other words, if SMA-proper is upstream of PMCd in the inhibitory pathway, then it might be possible that earlier (with respect to the STOP) stimulations could also influence behavior, as well as later stimulations, if SMA-proper is located downstream. Further experiments are required to investigate this possibility. Considering available data, there is evidence that invasive stimulation of the SMAproper and recordings from epileptic patients indicate that the output of cortical stimulation or the epileptic activity from SMA-proper may be of negative nature (Meletti et al., 2000; Krainik et al., 2001; Filevich et al., 2012). For instance, it has been suggested that the involvement of frontomesial areas in their epileptic patient could account for the negative motor events through a disruption of M1 output (Meletti et al., 2000). In epilepsy and tumor surgery, resection of the SMA-proper leads to transitory motor and speech deficits called the supplementary motor area syndrome (Krainik et al., 2001, 2003). This SMA syndrome is characterized by suppression or reduction in motor and speech production (Krainik et al., 2004). Furthermore, functional neuroimaging data showed SMAproper activity when people merely view graspable objects, without any intention to act upon them (Gre` zes and Decety, 2002). Such an activity has been interpreted in terms of an automatic inhibitory process concerning actions, which might be afforded by the viewed object but are in fact not required to be performed (see Nachev et al., 2008). This interpretation is consistent with observations in patients with microlesion of SMA-proper, who, different from healthy people and control patients with preSMA damage, are impaired in automatic suppression of evoked motor plans (Sumner et al., 2007). Conversely, the role of the SMA-proper in countermanding tasks is apparently limited, as shown by single-cell recordings from pre-SMA and SMA-proper neurons in monkeys. It was shown that only a very small percentage of recorded neurons (2.4\%) were involved in action inhibition (Scangos and Stuphorn, 2010). It is therefore plausible to hypothesize that SMA-proper is not one of the main actors in canceling a movement after the appearance of an external imperative STOP-signal. SMA-proper contribution is probably more prominent in self-initiated inhibitory mechanisms (Ridderinkhof et al., 2014) and inhibition of effectors during complex bimanual movements (Cincotta and Ziemann, 2008) rather than in inhibition of an impending movement in response to a contextual change. This is in line with the general physiological properties and connectivity pattern of SMAproper, which render it unlikely to support visuomotor transformations (Picard and Strick, 1996) that are required in our SST. Taken together, our findings indicate that there is a portion of PMCd playing a distinctive role in stopping instructed behavior on the basis of arbitrary external cues. This region could represent one of the putative lowest hierarchical nodes for action inhibition. We speculate that this finding could have potential implications in clinically relevant disorders of movement and of action control. We know that the SST paradigm in general has proven to be a useful tool for investigating response-inhibition deficits in psychopathological and neurological disorders - indeed some disorders, such as attention-deficit/hyperactivity disorder (as ADHD), compulsive disorders (as OCD, trichotillomania, and Tourette's syndrome), and substance-abuse disorders are described specifically as inhibitory disorders and consistently tested with the SST paradigm (Verbruggen and Logan, 2008). The portion of PMCd we examined in the present study could be a promising cortical area for the assessment and the manipulation of inhibitory capacities in the abovementioned neuropsychiatric disorders of self-control. It is also possible to hypothesize that the inhibitory control shown by the premotor cortex could be a basic mechanism in post-stroke reorganization and compensatory phases, both in the affected and in the nonaffected hemispheres. Although further research is required in this direction, an extension of the present result could be achieved in redesigning tasks devoted to the re-learning of skilled actions, as the one proposed in Dodakian and colleagues (2013). On the other side, different from PMCd, SMA-proper seems not be involved in 
stopping an ongoing action, following explicit instructions, in response to an external sensory cue. Nevertheless, the literature is clear in identifying the SMA-proper as a "negative motor area", i.e. a region that produces an interruption of voluntary movement when stimulated. We propose that premotor regions exert task-specific forms of inhibitory control. PMCd produces externally cued conditional inhibition. Hypothetically, the welldocumented inhibitory properties of SMA-proper could be related to automatic or internally generated inhibitory drives. Although further research is needed, we can speculate on the presence of a "mosaic" of inhibitory functions in the premotor cortices, with different premotor areas affecting movement according to the different task demands. Data obtained in humans during intraoperative cortical stimulation (Filevich et al., 2012) are also consistent with this hypothesis, indicating that negative motor areas are distributed multifocally in the premotor cortex, in a medial cluster (SMA-proper and pre-SMA) and a lateral cluster (IFG and premotor cortex). In the present study, we have shown that there is a portion of the lateral cluster involved in action inhibition, not only performed by the upper limbs but also by the lower face, when this inhibition occurs in response to an arbitrarily defined visuomotor association.

\section{CONFLICT OF INTEREST}

The authors declare no competing financial interests.

\section{ACKNOWLEDGMENTS}

None.

\section{REFERENCES}

Asmussen MJ, Jacobs MF, Lee KGH, Zapallow CM, Nelson AJ

(2013) Short-latency afferent inhibition modulation during finger movement. PLoS One:8.

Asmussen MJ, Zapallow CM, Jacobs MF, Lee KGH, Tsang P, Nelson AJ (2014) Modulation of short-latency afferent inhibition depends on digit and task-relevance. PLoS One:9.

Association WM (2009) Declaration of Helsinki. Ethical Principles for Medical Research Involving Human Subjects. Available at: http:// philpapers.org/rec/ASSDOH [Accessed September 3, 2015].

Badre D, D'Esposito M (2009) Is the rostro-caudal axis of the frontal lobe hierarchical? Nat Rev Neurosci 10:659669.

Baumgartner C, Podreka I, Olbrich A, Novak K, Serles W, Aull S, Almer G, Lurger S, Pietrzyk U, Prayer D, Lindinger G (1996) Epileptic negative myoclonus: An EEG-single-photon emission CT study indicating involvement of premotor cortex Available at:. Neurology 46:753-758. Available from: http://www.ncbi.nlm. nih.gov/pubmed/8618677.

Bizzi E, Tresch MC, Saltiel P, d'Avella A (2000) New perspectives on spinal motor systems Available at:. Nat Rev Neurosci 1:101-108.

Available from: http://www.ncbi.nlm.nih.gov/pubmed/11252772.

Caminiti R, Borra E, Visco-Comandini F, Battaglia-Mayer A, Averbeck BB, Luppino G (2017) Computational architecture of the parieto-frontal network underlying cognitive-motor control in monkeys ENEURO.030616.2017. Available at:. Eneuro 4. Available from: http://eneuro.sfn.org/lookup/doi/10.1523/ ENEURO.030616.2017.

Cincotta M, Ziemann U (2008) Neurophysiology of unimanual motor control and mirror movements. Clin Neurophysiol 119:744-762.

Dodakian L, Sharp KG, See J, Abidi NS, Mai K, Fling BW, Le VH, Cramer SC (2013) Targeted engagement of a dorsal premotor circuit in the treatment of post-stroke paresis. NeuroRehabilitation 33:13-24.

Dum RP, Strick PL (1991) The origin of corticospinal projections from the premotor areas in the frontal lobe. J Neurosci 11:667-689. 
Dum RP, Strick PL (2005) Frontal lobe inputs to the digit representations of the motor areas on the lateral surface of the hemisphere Available at:. J Neurosci 25:1375-1386. Available from: http://www.ncbi.nlm.nih.gov/pubmed/15703391.

Duque J, Greenhouse I, Labruna L, Ivry RB (2017) Physiological markers of motor inhibition during human behavior Available at:. Trends Neurosci 40:219-236. Available from: https://doi.org/10. 1016/j.tins.2017.02.006.

Duque J, Labruna L, Verset S, Olivier E, Ivry RB (2012) Dissociating the role of prefrontal and premotor cortices in controlling inhibitory mechanisms during motor preparation. J Neurosci 32:806-816.

Filevich E, Ku“ hn S, Haggard P (2012) Negative motor phenomena in cortical stimulation: Implications for inhibitory control of human action. Cortex 48:1251-1261.

Gerloff C, Corwell B, Chen R, Hallett M, Cohen LG (1997) Stimulation over the human supplementary motor area interferes with the organization of future elements in complex motor sequences. Brain 120:1587-1602.

Gre`zes J, Decety J (2002) Does visual perception of object afford action? Evidence from a neuroimaging study. Neuropsychologia 40:212-222.

Hamada M, Ugawa Y, Tsuji S (2008) High-frequency rTMS over the supplementary motor area for treatment of Parkinson's disease Available at:. Mov Disord 23:1524-1531. Available from: http://doi.wiley.com/10.1002/mds.22168.

Hamani C, Saint-Cyr JA, Fraser J, Kaplitt M, Lozano AM (2004) The subthalamic nucleus in the context of movement disorders. Brain 127:4-20.

Hatanaka N, Nambu A, Yamashita A, Takada M, Tokuno H (2001) Somatotopic arrangement and corticocortical inputs of the hindlimb region of the primary motor cortex in the macaque monkey. Neurosci Res 40:9-22.

Kalaska JF, Crammond DJ (1995) Deciding not to GO: neuronal correlates of response selection in a GO/NOGO task in primate premotor and parietal cortex. Cereb Cortex 5:410-428.

Kiefer M, Marzinzik F, Weisbrod M, Scherg M, Spitzer M (1998) The time course of brain activations during response inhibition: evidence from event-related potentials in a go/no go task. Neuroreport 9:765-770.

Koch G, Franca M, Del Olmo MF, Cheeran B, Milton R, Alvarez Sauco M, Rothwell JC (2006) Time course of functional connectivity between dorsal premotor and contralateral motor cortex during movement selection. J Neurosci 26:7452-7459. Available at: http://www.jneurosci.org/content/26/28/7452.short [Accessed June 15, 2015].

Krainik A, Duffau H, Capelle L, Cornu P, Boch A-L, Mangin J-F, Le Bihan D, Marsault C, Chiras J, Lehe' ricy S (2004) Role of the healthy hemisphere in recovery after resection of the supplementary motor area. Neurology 62:1323-1332.

Krainik A, Lehe' ricy S, Duffau H, Vlaicu M, Poupon F, Capelle L, Cornu P, Clemenceau S, Sahel M, Valery CA, Boch AL, Mangin JF, Bihan DL, Marsault C (2001) Role of the supplementary motor area in motor deficit following medial frontal lobe surgery. Neurology 57:871-878.

Krainik A, Lehericy S, Duffau H, Capelle L, Chainay H, Cornu P, Cohen L, Boch A-L, Mangin J-F, Le Bihan D, Marsault C (2003) Postoperative speech disorder after medial frontal surgery: role of the supplementary motor area Available at:. Neurology

60:587-594. Available from: http://www.neurology.org/cgi/doi/10. 1212/01.WNL.0000048206.07837.59.

Kroeger J, Ba“ umer T, Jonas M, Rothwell JC, Siebner HR, Mu“ nchau A (2010) Charting the excitability of premotor to motor connections while withholding or initiating a selected movement. Eur J Neurosci 32:17711779.

Ledberg A, Bressler SL, Ding M, Coppola R, Nakamura R (2007) Large-scale visuomotor integration in the cerebral cortex. Cereb Cortex 17:44-62. 
Li N, Daie K, Svoboda K, Druckmann S (2016) Robust neuronal dynamics in premotor cortex during motor planning Available at:. Nature 532:459-464. Available from: http://www.nature.com/ doifinder/10.1038/nature17643.

Lu“ ders $\mathrm{H}$ et al (1998) Semiological seizure classification. Epilepsia 39:1006-1013.

Mayka MA, Corcos DM, Leurgans SE, Vaillancourt DE (2006) Threedimensional locations and boundaries of motor and premotor cortices as defined by functional brain imaging: a meta-analysis. Neuroimage 31:14531474.

Meletti S, Tinuper P, Bisulli F, Santucci M (2000) Epileptic negative myoclonus and brief asymmetric tonic seizures. A supplementary sensorimotor area involvement for both negative and positive motor phenomena. Epileptic Disord 2:163-167.

Mikuni N, Ohara S, Ikeda A, Hayashi N, Nishida N, Taki J, Enatsu R, Matsumoto R, Shibasaki H, Hashimoto N (2006) Evidence for a wide distribution of negative motor areas in the perirolandic cortex. Clin Neurophysiol 117:33-40.

Mirabella G, Pani P, Ferraina S (2011) Neural correlates of cognitive control of reaching movements in the dorsal premotor cortex of rhesus monkeys. J Neurophysiol 106:1454-1466.

Mirdamadi JL, Suzuki LY, Meehan SK (2017) Attention modulates specific motor cortical circuits recruited by transcranial magnetic stimulation Available at:. Neuroscience 359:151-158. Available from: https://doi.org/10.1016/j.neuroscience.2017.07.028.

Moll L, Kuypers HG (1977) Premotor cortical ablations in monkeys: contralateral changes in visually guided reaching behavior. Science 198:317-319.

Muakkassa KF, Strick PL (1979) Frontal lobe inputs to primate motor cortex: evidence for four somatotopically organized "premotor" areas. Brain Res 177:176-182.

Nachev P, Kennard C, Husain M (2008) Functional role of the supplementary and pre-supplementary motor areas. Nature Reviews Neuroscience 9(11):856.

Nii Y, Uematsu S, Lesser RP, Gordon B (1996) Does the central sulcus divide motor and sensory functions? Cortical mapping of human hand areas as revealed by electrical stimulation through subdural grid electrodes. Neurology 46:360-367.

Noorani I, Carpenter RHS (2017) Not moving: the fundamental but neglected motor function Available at:. Philos Trans R Soc B Biol Sci 372:20160190. Available from: http://rstb. royalsocietypublishing.org/lookup/doi/10.1098/rstb.2016.0190.

Obeso I, Cho SS, Antonelli F, Houle S, Jahanshahi M, Ko JH, Strafella AP (2013) Stimulation of the pre-SMA influences cerebral blood flow in frontal areas involved with inhibitory control of action. Brain Stimul 6:769776.

Parmigiani S, Barchiesi G, Cattaneo L (2015) The dorsal premotor cortex exerts a powerful and specific inhibitory effect on the ipsilateral corticofacial system: a dual-coil transcranial magnetic stimulation study Available at:. Exp Brain Res. Available from: http://link.springer.com/10.1007/s00221-015-4393-7.

Parmigiani S, Zattera B, Barchiesi G, Cattaneo L (2018) Spatial and temporal characteristics of set-related inhibitory and excitatory inputs from the dorsal premotor cortex to the ipsilateral motor cortex assessed by dual-coil transcranial magnetic stimulation. Brain Topogr.

Picard N, Strick PL (1996) Motor areas of the medial wall: a review of their location and functional activation. Cereb Cortex 6:342-353.

Picton TW, Stuss DT, Alexander MP, Shallice T, Binns MA, Gillingham S (2007) Effects of focal frontal lesions on response inhibition Available at:. Cereb Cortex 17:826-838. Available from: http://www.cercor.oxfordjournals.org/cgi/doi/10.1093/cercor/ bhk031.

Ridderinkhof KR, van den Wildenberg WPM, Brass M (2014) "Don't" versus "Won't": principles, mechanisms, and intention in action inhibition Available at:. Neuropsychologia 65:255-262. Available from: 
https://doi.org/10.1016/j.neuropsychologia.2014.09.005. Rizzolatti G, Luppino G (2001) The cortical motor system. Neuron 31:889-901.

Rossi S, Hallett M (2009) Safety, ethical considerations, and application guidelines for the use of transcranial magnetic stimulation in clinical practice and research Available at:. Clin Neurophysiol 120:2008-2039. Available from: http:// www.sciencedirect.com/science/article/pii/S1388245709005197.

Sawaguchi T, Yamane I, Kubota K (1996) Application of the GABA antagonist bicuculline to the premotor cortex reduces the ability to withhold reaching movements by well-trained monkeys in visually guided reaching task. J Neurophysiol 75:2150-2156.

Scangos KW, Stuphorn V (2010) Medial frontal cortex motivates but does not control movement initiation in the countermandering task. J Neurosci 30:1968-1982.

Sumner P, Nachev P, Morris P, Peters AM, Jackson SR, Kennard C, Husain M (2007) Human medial frontal cortex mediates unconscious inhibition of voluntary action. Neuron 54:697-711.

Tokuno H, Tanji J (1993) Input organization of distal and proximal forelimb areas in the monkey primary motor cortex: a retrograde double labeling study. J Comp Neurol 333:199-209.

Verbruggen F, Logan G (2008) Response inhibition in the stop-signal pardigm Available at:. Trends Cogn Sci 12:418-424. Available from: http://www.sciencedirect.com/science/article/pii/ S1364661308002155.

Voller B, St Clair Gibson A, Dambrosia J, Pirio Richardson S, Lomarev M, Dang N, Hallett M (2006) Short-latency afferent inhibition during selective finger movement. Exp Brain Res 169:226-231.

Vorobiev V, Govoni P, Rizzolatti G, Matelli M, Luppino G (1998) Parcellation of human mesial area 6: cytoarchitectonic evidence for three separate areas. European Journal of Neuroscience 10(6):2199-2203. https://doi.org/10.1046/i.1460-9568.1998.

00236.x. 